\title{
ON PERFORMANCE ANALYSIS OF CDMA UNSLOTTED ALOHA SYSTEM WITH BURST RESERVATION
}

\author{
Jae-Woo So, Il Han, Byung-Cheol Shin, and Dong-Ho Cho \\ Department of Electrical Engineering \\ Korea Advanced Institute of Science and Technology, Taejon 305-701, Korea \\ PH: +82-42-869-8067, FAX: +82-42-867-0449 \\ e-mail: \{israin, ilhan\}@comis.kaist.ac.kr, bcshin@cbucc.chungbuk.ac.kr, \\ dhcho@ee.kaist.ac.kr
}

\begin{abstract}
In this paper, we analyze the performance of CDMA unslotted ALOHA system with burst reservation. A mobile terminal reserves the channel for the duration of its burst and transmits spread packets. The packets are transmitted in unslotted mode. As the packet transmission of mobile terminal can be begun at any time, the level of multi-user interference fluctuates during the packet transmission. We, therefore, calculate the bit error probability and throughput by considering not only the number of overlapped packets but also the amount of time overlap. We assume that the generation of bursts is Poisson distributed, the number of packets transmitted at once by a mobile terminal is geometrically distributed, and the packet length is constant. By modeling the system as a Markov chain, we obtain the throughput of CDMA unslotted ALOHA system with burst reservation.
\end{abstract}

\section{INTRODUCTION}

Currently, the packet radio systems based on the code division multiple access (CDMA) have received significant attention as a system for third generation mobile communication because of features such as random access capability, potentiality for high throughput performance, and low peak power in the transmission [1]. The packet radio systems based on CDMA can be classified into two systems, CDMA slotted ALOHA system and CDMA unslotted ALOHA system according to access schemes. In CDMA ALOHA system with burst reservation, a mobile terminal reserves the channel for the duration of its burst and transmits spread packets based on methods similar to other reservation-based protocols such as Packet Reservation Multiple Access (PRMA), PRMA++, and PRMA/CDMA [2]. In
CDMA slotted ALOHA, packet transmission is initiated only at the beginning of a slot and the success of packet depends on the number of interference within the slot. In contrast to CDMA slotted ALOHA, the packet in CDMA unslotted ALOHA can be transmitted at any time, so it does not require synchronization. Its system implementation, therefore, is easier than CDMA slotted ALOHA. However, the analysis of CDMA unslotted ALOHA is more difficult because the level of user interference fluctuates during the packet transmission.

Many people have studied on the analysis and improvements of the CDMA ALOHA systems. Yin and $\mathrm{Li}[3]$ introduced a new technique to analyze the throughput of an $L$-channel CDMA network. Their analysis of the unslotted CDMA system with fixed packet length was based on the ballot theory considering the level of signal to noise ratio during packet transmission. Toshimitsh et al. [4] presented a novel spread slotted ALOHA system with a CLSP. The slot size of the proposed system was assumed to be less than the packet size. They, therefore, were able to apply CLSP and improved the throughput of spread slotted ALOHA system. Sato et al. [5] analyzed the CDMA unslotted ALOHA system with fixed packet length. As the levels of multiuser interference fluctuate during the packet transmission, they calculated the packet success probability and throughput.

In this paper, we analyze the performance of CDMA unslotted ALOHA system with burst reservation. A mobile terminal can transmit its burst in unslotted mode after receiving the permission granted packet and spread sequence from hub station. The hub station senses the channel load, which means the number of simultaneous transmission, and controls the

0-7803-5565-2/99/\$10.00 두 1999 IEEE 
permission of mobile terminal's transmission so as not to cause the severe multiuser interference. This paper is organized as follows: In Section II, the system model of CDMA unslotted ALOHA system with burst reservation is presented. The system parameters such as packet success probability and throughput are analyzed in Section III. In Section IV, numerical results and computer simulation results are compared, and concluding remarks are presented in Section V.

\section{SYSTEM DESCRIPTION AND MODELING}

A mobile terminal sends a reservation request packet to hub station for burst transmission and waits for permission. Hub station senses the channel load and then sends a permission granted packet to mobile terminal if the channel load is less than or equal to a certain threshold $\alpha$. This scheme is similar to CLSP (Channel Load Sensing Protocol) [6]. The mobile terminal receiving the permission granted packet transmits its burst at any bit time. The state transition diagram is shown in Fig. 1.

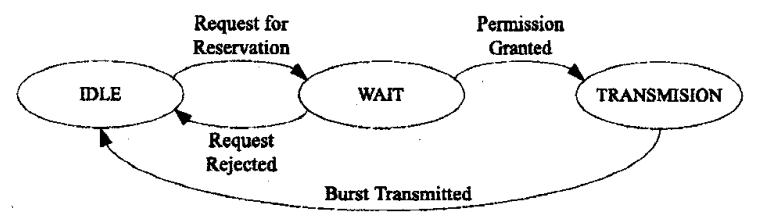

Figure 1: State transition diagram

To evaluate the throughput performance of the CDMA unslotted ALOHA system with burst reservation, we have a few assumptions as follows: (1) The generation of bursts is assumed to be Poisson distribution with arrival rate $\lambda$. (2) The number of packets transmitted during a burst by a mobile terminal is geometrically distributed with average number of packets $\bar{B}$ and a packet length is constant $L$. (3) Every transmitted packets are received with equal power. (4) Bit errors in a packet are caused by the effect of multiple access interference and additive white Gaussian noise (AWGN) [5]. The bit error probability is expressed as [7]

$$
\begin{aligned}
P_{b}(k)= & \frac{2}{3} Q\left[\left(\frac{k}{3 N}+\frac{N_{0}}{2 E_{b}}\right)^{-0.5}\right] \\
& +\frac{1}{6} Q\left[\left(\frac{k(N / 3)+\sqrt{3} \sigma}{N^{2}}+\frac{N_{0}}{2 E_{b}}\right)^{-0.5}\right] \\
& +\frac{1}{6} Q\left[\left(\frac{k(N / 3)-\sqrt{3} \sigma}{N^{2}}+\frac{N_{0}}{2 E_{b}}\right)^{-0.5}\right]
\end{aligned}
$$

where

$$
\sigma^{2}=k\left[N^{2} \frac{23}{360}+N\left(\frac{1}{20}+\frac{k-1}{36}\right)-\frac{1}{20}-\frac{k-1}{36}\right] .
$$

Here, $N$ is the number of chip per bit, $k$ is the number of interfering packet, $N_{0}$ is the two-sided spectral density of Gaussian noise, and $Q(x)$ is given by

$$
Q(x)=\frac{1}{\sqrt{2 \pi}} \int_{x}^{\infty} e^{-u^{2} / 2} d u
$$

\section{THROUGHPUT ANALYSIS}

\section{Transition of the Number of Interfer- ing Packets}

The number of interference level varies during its burst because mobile terminals can transmit their bursts at any time. For calculating a packet success probability, we suppose "tagged" packet as shown in Fig. 2 and arrange the other messages in order for simplicity. This figure shows that the number of interfering packets varies while the tagged packet is transmitted.

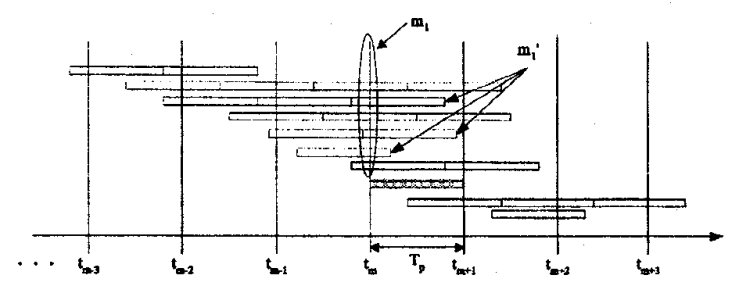

Figure 2: A fluctuation of interfering level during tagged packet transmission

Because the generation of arriving bursts is Poisson distribution, the probability that $k$ bursts will enter the network in a packet duration $T_{p}$ is given by

$$
P(k)=\frac{\left(\lambda T_{p}\right)^{k}}{k !} e^{-\lambda T_{p}}
$$

Now, we calculate the probability $P(m)$ that there are $m$ packets at a certain time $t_{m}$. First, we assume that the number of bursts that arrive in time interval $\left[t_{m-i}, t_{m-i+1}\right)$ and depart channel after $t_{m}$ is equal to $a_{i}$. Then, $P\left(a_{i}\right)$ is calculated as following.

$$
\begin{aligned}
P\left(a_{i}\right) & =\sum_{k=a_{i}}^{\infty} P\left(a_{i} \mid k\right) P(k) \\
& =\sum_{k=a_{i}}^{\infty}\left(\begin{array}{c}
k \\
a_{i}
\end{array}\right) P(B \geq i)^{a_{i}}(1-P(B \geq i))^{k-a_{i}} P(k)
\end{aligned}
$$


where the probability $P(B \geq i)$ that the number of packets in a burst is larger than $i$ packets is calculated as follows.

$P(B \geq i)=\sum_{x=i}^{\infty} p \cdot(1-p)^{x-1}=q^{i-1}, q=1-p(6)$

Let a time interval $\left[t_{m-i}, t_{m-i+1}\right)$ be interval $i$ and we define that the number of bursts that arrive in (interval 1 , interval $2, \cdots$, interval $M$ ) and depart channel after $t_{m}$ is equal to $\left(a_{1}, a_{2}, \cdots, a_{M}\right)$, respectively. As the probabilities $P\left(a_{i}\right)$ s are independent, the probability $P\left(a_{1}, a_{2}, \cdots, a_{M}\right)$ is obtained by mutual products as following.

$$
P\left(a_{1}, a_{2}, \cdots, a_{M}\right)=P\left(a_{1}\right) P\left(a_{2}\right) \cdots P\left(a_{M}\right)
$$

where $M$ is the maximum number of packets could be transmitted at once by a mobile terminal. Accordingly, the probability $P(m)$ is given by

$$
\begin{array}{r}
P(m)=\sum_{a_{1}} \sum_{a_{2}} \cdots \sum_{a_{M}} P\left(a_{1}, a_{2}, \cdots, a_{M}\right) \\
, a_{1}+a_{2}+\cdots+a_{M}=m .
\end{array}
$$

\section{System Offered Load}

Offered load $G$ is defined as the average number of generating packets during packet duration $T_{p}$ and can be expressed as

$$
G=\lambda \cdot T_{p} \cdot \bar{B}
$$

where $T_{p}$ is a packet duration, i.e., $T_{p}=L / R$ and $L$ [bits] is the length of a packet. Also $R[\mathrm{~b} / \mathrm{s}]$ is the data rate and $B$ is the average number of packets transmitted at once. Because the number of ongoing packets on channel is always less than or equal to $\alpha$, the system offered load $G_{\text {sys }}$ is given by

$$
G_{\text {sys }}=\frac{\sum_{m=0}^{\alpha} m \cdot P(m)}{\sum_{m=0}^{\alpha} P(m)}
$$

where $m$ is the number of packets and the probability $P(m)$ is obtained by (8). Furthermore, we define the normalized offered load as

$$
G_{n o r m}=\frac{G}{N} \cdot \frac{L}{T_{p}} \quad[\mathrm{bits} / \mathrm{Hz} / \mathrm{sec}]
$$

where $N$ is spreading factor. Because the number of packets is geometrically distributed with average number of packets $\bar{B}$, the probability that the number of packets transmitted at once is $x$ [packets] is given by

$$
P(B=x)=p \cdot(1-p)^{x-1}, \quad p=1 / \bar{B} .
$$

\section{Derivation of Conditional State Tran- sition Probability}

If the number of interfering packets is $m_{i}$ on $i$ th bit, the interfering packets increase to $m_{i}+1$, decrease to $m_{i}-1$, or remain to $m_{i}$ during bit timing since the number of interference level is varying bit by bit during the tagged packet transmission. The number of interfering packets is always less than $\alpha$ since the number of ongoing packets is controlled below a certain threshold $\alpha$ by hub station. Let the bit timing be $\Delta t$, then the system can be modeled by Markov chain as shown in Fig. 3.

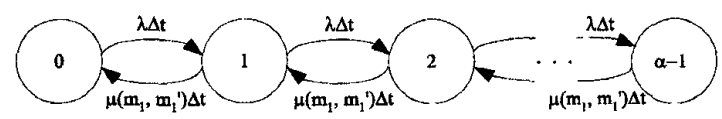

Figure 3: State transition of the number of interfering packets

If there are $m_{1}$ bursts on first bit of tagged packet and the number of bursts that depart during the packet duration $T_{p}$ among $m_{1}$ bursts is equal to $m_{1}^{\prime}$ as shown in Fig 2, the average service time of $m_{1}^{\prime}$ is $T_{p} / m_{1}^{\prime}$ and death rate $\mu\left(m_{1}, m_{1}^{\prime}\right)$ is derived as [5]

$$
\mu\left(m_{1}, m_{1}^{\prime}\right)=\frac{m_{1}^{\prime}}{T_{p}} .
$$

Also, birth rate $\lambda$ is obtained by (9).

$$
\lambda=\frac{G}{T_{p} \cdot \bar{B}}
$$

Accordingly, the conditional state transition probability is expressed as

$$
\begin{aligned}
& q\left(m_{j} \mid m_{j-1}\right) \\
& =\left\{\begin{array}{cl}
1-\lambda \Delta t-\mu\left(m_{1}, m_{1}^{\prime}\right) \Delta t \\
, \text { if } m_{j}=m_{j-1}, m_{j}<\alpha-1 \\
1-\mu\left(m_{1}, m_{1}^{\prime}\right) \Delta t \\
, \text { if } m_{j}=m_{j-1}, m_{j}=\alpha-1 \\
\mu\left(m_{1}, m_{1}^{\prime}\right) \Delta t \\
\quad, \text { if } m_{j}=m_{j-1}-1, m_{j}<\alpha-1 \\
\lambda \Delta t \quad \text { if } m_{j}=m_{j-1}+1, m_{j} \leq \alpha-1 \\
0 \quad, \text { otherwise. }
\end{array}\right.
\end{aligned}
$$

\section{Derivation of Packet Success Probabil- ity}

To calculate the packet success probability, we define an extended function $f_{j}\left(m_{j}, m_{1}, m_{1}^{\prime}\right)$ used in 
Ref. [5]. The tagged packet is transmitted successfully from the first bit to the $(j-1)$ th bit, where $m_{1}^{\prime}$ bursts depart during packet duration $T_{p}$ among $m_{1}$ bursts which is the number of interfering bursts on first bit. We can calculate the function $f_{j}\left(m_{j}, m_{1}, m_{1}^{\prime}\right)$ recursively based on Markovian property of $m_{j}$. First, when $j$ is the first bit of the tagged packet, $f_{j}\left(m_{j}, m_{1}, m_{1}^{\prime}\right)$ is the function of probability $P\left(m_{1}^{\prime}, m_{1}\right)$.

$$
f_{j}\left(m_{j}, m_{1}, m_{1}^{\prime}\right)=\frac{P\left(m_{1}, m_{1}^{\prime}\right)}{\sum_{m_{1}=0}^{\alpha-1} P\left(m_{1}\right)}
$$

where $P\left(m_{1}\right)$ is obtained by $(8)$ and $P\left(m_{1}, m_{1}^{\prime}\right)$ is calculated as follows.

$$
\begin{aligned}
& P\left(m_{1}, m_{1}^{\prime}\right) \\
& \quad=P\left(m_{1}\right) P\left(m_{1}^{\prime} \mid m_{1}\right) \\
& =P\left(m_{1}\right)\left(\begin{array}{c}
m_{1} \\
m_{1}^{\prime}
\end{array}\right)\left(\frac{P(B=i)}{P(B \geq i)}\right)^{m_{1}^{\prime}}\left(1-\frac{P(B=i)}{P(B \geq i)}\right)^{m_{1}-m_{1}^{\prime}} \\
& \quad=P\left(m_{1}\right)\left(\begin{array}{c}
m_{1} \\
m_{1}^{\prime}
\end{array}\right) p^{m_{1}^{\prime}(1-p)^{m_{1}-m_{1}^{\prime}}} \quad, i=1,2,3, \cdots
\end{aligned}
$$

When $j$ is not the first bit of the tagged packet, $f_{j}\left(m_{j}, m_{1}, m_{1}^{\prime}\right)$ is calculated recursively as following.

$$
\begin{aligned}
& f_{j}\left(m_{j}, m_{1}, m_{1}^{\prime}\right)=\sum_{m_{j-1}=m_{j}-1}^{m_{j}+1}\left[f_{j-1}\left(m_{j-1}, m_{1}, m_{1}^{\prime}\right)\right. \\
&\left.\cdot q\left(m_{j} \mid m_{j-1}\right) \cdot\left(1-P_{b}\left(m_{j-1}\right)\right)\right]
\end{aligned}
$$

Using $f_{j}\left(m_{j}, m_{1}, m_{1}^{\prime}\right)$, we can calculate the packet success probability $Q_{s}$ recursively. Because the length of packet is constant $L$, the packet success probability is calculated by setting $j=L$.

$$
\begin{array}{r}
Q_{s}=\sum_{m_{L}=0}^{\alpha-1} \sum_{m_{1}=0}^{\alpha-1} \sum_{m_{1}^{\prime}=0}^{m_{1}}\left[f_{L}\left(m_{L}, m_{1}, m_{1}^{\prime}\right)\right. \\
\left.\cdot\left(1-P_{b}(m)\right)\right]
\end{array}
$$

On the other hand, the system throughput is defined as the average number of successful transmissions in a packet duration $T_{p}$. Thus, the system throughput $S_{s y s}$ and normalized throughput $S_{\text {norm }}$ are obtained as

$$
\begin{aligned}
S_{\text {sys }} & =G \cdot Q_{s} \\
S_{\text {norm }} & =\frac{S}{N} \cdot \frac{L}{T_{p}} \quad[\mathrm{bits} / \mathrm{Hz} / \mathrm{sec}]
\end{aligned}
$$

\section{NUMERICAL AND SIMULA- TION RESULTS}

In this section, we compare the numerical results with simulation results of CDMA unslotted ALOHA system with burst reservation. Bit errors in a packet are assumed to be caused by multiuser interference and additive white Gaussian noise. The system parameters used in this simulation are summarized in Table 1 .

Table 1: System Prameters

\begin{tabular}{lll}
\hline \hline Item & Symbol & Value \\
\hline \hline Data rate (kbps) & $R$ & 9.6 \\
\hline Spreading factor & $N$ & 30 \\
\hline $\begin{array}{l}\text { Average length of a burst } \\
\text { (packets) }\end{array}$ & $\bar{B}$ & 5 \\
\hline Packet length (bits) & $L$ & 512 \\
\hline Threshold of channel load & $\alpha$ & variable \\
\hline \hline
\end{tabular}

First, when hub station don't control channel load, that is, the threshold of channel load $\alpha$ is infinity, throughput performance for various $E_{b} / N_{0}$ is shown in Fig. 4. Infinity value of $E_{b} / N_{0}$ means that the effect of additive white Gaussian noise is neglected and so bit errors can occur due to multiuser interference. As expected, results show that the throughput of CDMA unslotted ALOHA system with burst reservation also exhibits the ALOHA-like throughput $(S)$ versus offered channel load $(G) . S$ increases with $G$, but eventually decreases as $G$ becomes larger. In Fig. 5 and Fig. 6, throughput performance of CDMA unslotted ALOHA system with burst reservation is shown for various threshold of channel load $\alpha$ when hub station controls channel load. Here, only multiuser interference is considered. For $\alpha<8$, throughput is lower than that of system without control of channel load because of over rejection of hub station. When $\alpha=8$, maximum throughput is obtained. The throughput decreases as $\alpha$ is larger than 8 and the same throughput as system without control of channel load is shown for $\alpha \geq 26$. These results imply that the setting of optimum threshold value of channel load is important.

\section{CONCLUSIONS}

Throughput of CDMA unslotted ALOHA system with burst reservation is analyzed in this paper. In this system, a mobile terminal reserves the channel for the duration of its burst and transmits spread packets in unslotted mode. Under assumption that the generation of bursts is Poisson distributed and the number of packets in a burst is geometrically distributed, the system can be modeled as a Markov chain. We obtain the throughput of system considering the variance of multi-user interference during a burst transmission. The results show that 


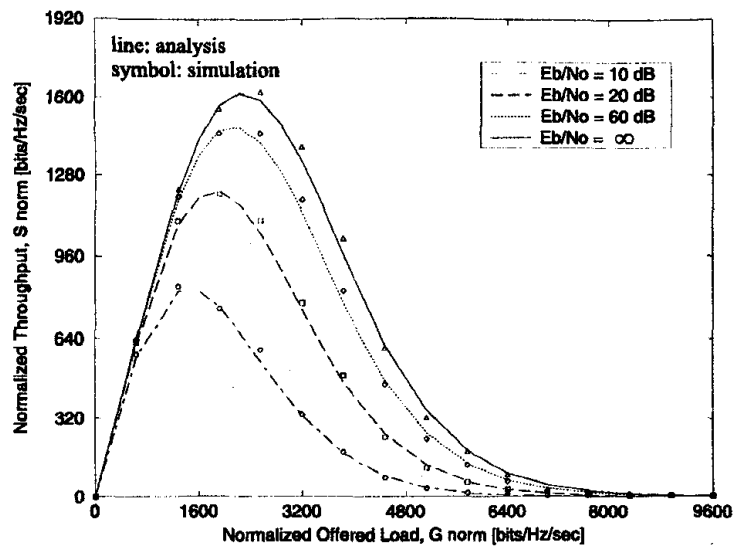

Figure 4: Throughput performance vs normalized offered load for various $E_{b} / N_{0}$ values

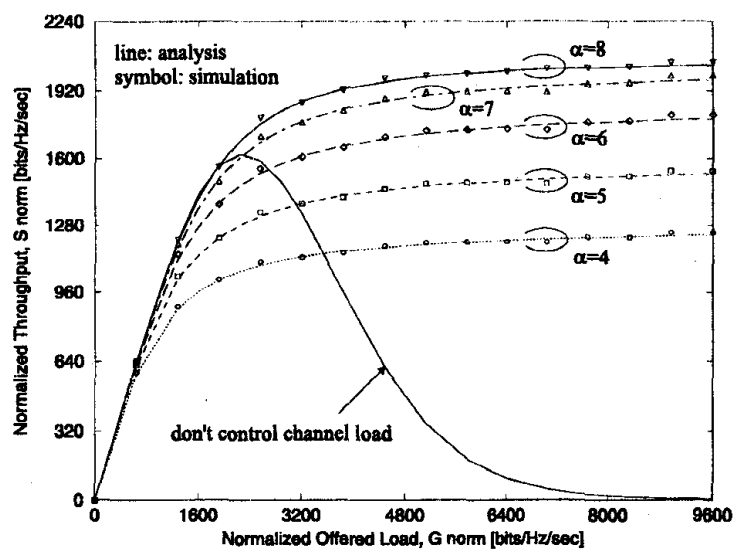

Figure 5: Throughput performance vs normalized offered load for various $\alpha$ values $(\alpha \leq 8)$

the throughput of CDMA unslotted ALOHA system with burst reservation exhibits the ALOHAlike throughput versus offered load when hub station doesn't control channel load. However, when hub station controls the level of multi-user interference, the system keeps high throughput at high load traffic. Furthermore, optimal threshold to maximize the number of simultaneous transmission of packets is obtained.

\section{REFERENCES}

(1) TIA TR45.5, The cdma2000 RTT Candidate Submissin to ITU-R, Draft Document, June 1998.

(2) A. E. Brand and A. H. Aghvami, "Performance of a joint CDMA/PRMA protocol for mixed

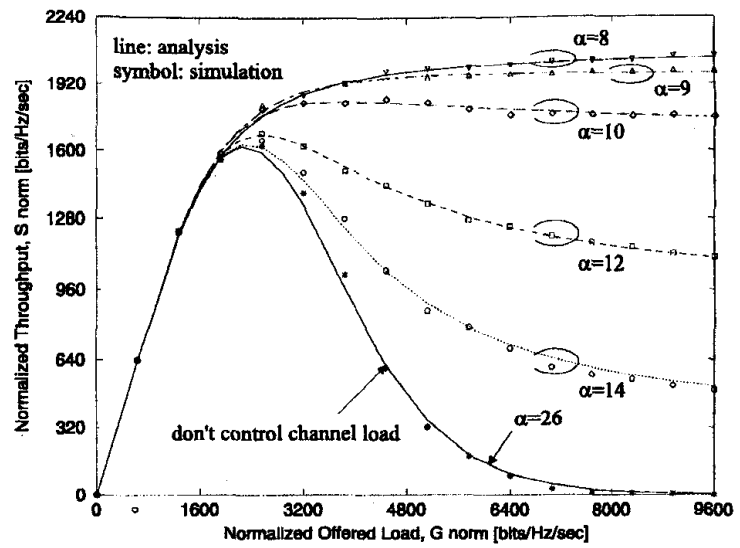

Figure 6: Throughput performance vs normalized offered load for various $\alpha$ values $(\alpha \geq 8)$

voice data transmission for third generation mobile communicatin", IEEE J. Select. Areas Commun., vol. 14, no. 9, pp. 1698-1707, December 1996.

(3) Ming Yin, and Victor O.K. Li, "Unslotted CDMA with Fixed Packet Length", IEEE $J$. Select. Areas in Commun., vol. 8, no. 4, pp. 529-541, May 1990

(4) Kiyoshi Toshimitsu, Takaya Yamazato, Masaaki Katayama, and Akira Ogawa, "A Novel Spread Slotted Aloha System with Channel Load Sensing Protocol", IEEE J. Select. Areas in Commun., vol. 12, no. 4, pp. 665-672, May 1994

(5) Takeshi Sato, Hiraku Okada, Takaya Yamazato, Massaki Katayama, and Akira Ogawa, "Throughput Analysis of DS/SSMA Unslotted ALOHA System with Fixed Packet Length", IEEE J. Select. Areas in Commun., vol. 14, no. 4, pp. 750-756, May 1996.

(6) A.H. Abdelmonem and T.N. Saadawi, "Performance Analysis of Spread Spectrum Packet Radio of Spread Spectrum Packet Radio Networks with Channel Load Sensing", IEEE J. Select. Areas in Commun., vol. 7, no. 4, pp. 161-166, January 1989.

(7) Jack M. Holtzman, "A Simple, Accurate Method to Calculate Spread-Spectrurm Multiple-Access Error Probabilities", IEEE Trans. Commun., vol. 40, no. 3, pp. 461-464, March 1992. 